\title{
IMPACTO MUNDIAL PSICOLÓGICO DA PANDEMIA CORONAVÍRUS EM DENTISTAS NA PRÁTICA CLÍNICA PRIVADA
}

\author{
PSYCHOLOGICAL IMPACT OF THE CORONAVIRUS PANDEMIC ON DENTISTS IN \\ WORLDWIDE CLINICAL PRIVATE PRACTIVE
}

\author{
Jôobeya Coutinho ${ }^{1}$ \\ Francielly Censi Boehm² \\ Cintia Aparecida Santana Avelino ${ }^{3}$ \\ Maria Vanderlandia Mendes Bezerra Aquino ${ }^{4}$ \\ Tatiane Cristina Albino Gomes ${ }^{5}$ \\ Clara Cavalero ${ }^{6}$ \\ Caio Junji Tanaka ${ }^{7}$
}

RESUMO: Os estados de emergências na saúde pública têm um impacto sobre a saúde mental da população. O surto do novo coronavírus (COVID-19) afetou o mundo todo de forma psicológica, economica, educacional e política. O objetivo deste estudo foi relatar questões que envolvem os impactos psicológicos nos cirugiões dentistas em contextos pessoais, familiares, financeiros e profissionais, de modo a compreender a nova realidade durante a pandemia da COVID-19. A revisão abrangeu toda a literatura até agosto de 2021; sendo incluídos estudos mais relevantes. Com base na revisão da literatura, pode-se concluir que o profissional da odontologia teve grande impacto na vida pessoal e profissional, sendo abalados emocionalmente desde o início da pandemia até os dias atuais. A resiliência, o autocuidado e a interação com profissional da mesma área é fundamental neste momento para que o dentista estabeleça estratégias e permaneça atuante nos atendimentos odontológicos de seus pacientes. $\mathrm{O}$ aconselhamento psicológico profissional lhe dará a liberdade de expor suas angústias, medos e preocupações em meio à pandemia que o mundo está vivendo.

Palavras-chave: Odontologia. Saúde mental. Pandemia. Covid-ı9.

ABSTRACT: Public health emergencies have a great impact on the mental health of the population. The coronavirus outbreak (COVID-I9) has affected the entire world psychologically, economically, educationally and politically. The aim of this study was to report issues involving the psychological impacts on dentists in personal, family, financial and professional contexts, in order to understand the new reality during the COVID-I9 pandemic. The review covered all available literature up to August 2021; the most relevant studies being included. Based on the

\footnotetext{
${ }^{I}$ Acadêmica do curso de graduação em psicologia da UNIVERITAS - campus Itaquaquecetuba/SP.

${ }^{2}$ Acadêmica do curso de graduação em odontologia da Universidade de Cuiabá - UNIC - campus Rondonópolis/MT. E-mail: franciellyboehm@hotmail.com.

${ }^{3}$ Acadêmica do curso de graduação em psicologia da UNIVERITAS - campus Itaquaquecetuba/SP.

${ }^{4}$ Acadêmica do curso de graduação em psicologia da UNIVERITAS - campus Itaquaquecetuba/SP.

${ }^{5}$ Acadêmica do curso de graduação em psicologia da UNIVERITAS - campus Itaquaquecetuba/SP.

${ }^{6}$ Formação em psicologia pela Universidade de Guarulhos/SP, Psicóloga do CER-Centro Especializado em Reabilitação da Prefeitura de São Paulo/SP, especialista em Neuropsicologia pelo HCFMUSP, formação em Reabilitação Cognitiva pela mesma instituição.

${ }^{7} \mathrm{PhD}$ em odontologia pela Universidade de Guarulhos/SP, Professor titular dos cursos de graduação em saúde da UNIVERITAS - campus Itaquaquaquecetuba/SP.
} 
literature review, it can be concluded that the dental professional had a great impact on personal and professional life, being emotionally shaken from the beginning of the pandemic to the present day. Resilience, self-care and interaction with a professional in the same area is essential at this time for the professional to establish strategies and remain active in the dental care of their patients. Professional psychological counseling will give you the freedom to expose your anxieties, fears and concerns in the midst of the pandemic that the world is experiencing.

keywords: Dentistry. Mental health. Pandemic. Covid-ı.

\section{INTRODUÇÃO}

A rápida dissipação da doença infectocontagiosa Sars-Cov2 desde o final de 2019 causou grandes impactos e mudanças na vida pessoal e profissional de toda a população mundial (Mulugeta et al. 2021). Assim como todos os profissionais da saúde, os dentistas foram muito afetados nesta pandemia, estudos descrevem que diante ao cenário atual, os profissionais da saúde possuem maior risco de desenvolverem doenças mentais (Buselli et al., n.d.).

O dentista, em sua prática clínica ododntológica, apresenta alta vulnerabilidade de infecção cruzada profissional-dentista, podendo ser superior a outros profissões da área da saúde (Coulthard 2020). Existe a grande preocupação nos atendimentos a pacientes présintomáticos e/ou assintomáticos, pelo fato de que o pico de transmissibilidade supostamente acontece entre I a 2 dias antes da manifestação dos sintomas da doença coronavírus (Meyerowitz et al. 2021).

Diante disso, a nova realidade de vida trouxe uma explosão de emoções e sentimentos, como medo da doença, síndrome de burnout, pânico, ansiedades, distúrbios do sono e outros transtornos mentais. Para muitos, estes impactos emocionais nocivos tornaram-se companheiros diários nesta longa jornada de pandemia (Bryant-genevier et al. 2021; Maunder 2004).

Em dezembro de 2019, foi registrado o primeiro caso de Sars-CoV-2, ocorrido em Wuhan na China, sendo um vírus letal. Esta doença não possui tratamento específico, as terapêuticas são realizadas conforme os sintomas apresentado pelo paciente (Khan and Khan 2021). Devido ao avanço da medicina e dos recursos tecnológicos, foi possível em tempo record, comparado a pandemias anteriores, a elaboração das vacinas (Coulthard 2020).

A contaminação se dá através das vias respiratórias, por meio de gotículas de saliva, ao falar, tossir e/ou espirrar, quando estamos próximo de pessoas contaminadas, bem 
como pelo toque nas mãos e pelo contato com objetos infectados. Por isso a importância dos cuidados com a higienização das mãos e uso de máscaras (Marqu and Domingo 2021).

O profissional dentário, um DDS - médico de cirurgia dentária, tem como missão principal os cuidados da saúde oral e devido a isto trabalha muito próximo a boca do paciente (Nemeth et al. 202I). Além de ter contato com a saliva, sangue e fluidos orais, os dentistas utilizam equipamentos que produzem aerossol, como por exemplo uma peça de mão de alta velocidade. Estes aerossóis podem resultar na propagação de microorganismos no ar (Passarelli et al. 2020). Estudos relatam que ainda não se sabe ao certo se os aerossóis produzidos pelos atendimentos odontológicos possuem transmissibilidade do SARS-CoV2 (Levit and Levit 2021; Innes et al. 2020).

No primeiro trimestre de 2020 a pandemia do Covid-ıg havia tomado a proporção global e as recomendações foram o fechamento dos consultórios odontológicos, pelo fato do alto risco de contaminação dentista-paciente. Os atendimentos então se restringiram aos pacientes em condições emergenciais de saúde bucal (Amato et al. 2020).

Após um curto período de tempo foi liberada a abertura dos consultórios, mas com uma anamnese/triagem dos pacientes e protocolos de biossegurança mais rigorosos (Derruau et al. 202I).

Para reduzir o risco de contaminação, os DDS devem utilizar de forma correta os equipamentos de proteção individual e além disso, manter uma limpeza rigorosa e contínua do local de trabalho, deve-se higienizar os objetos não descartáveis e se atentar para a correta lavagens das mãos (Villani et al. 2020). Estes protocolos já existentes na área da saúde, ganharam um rigor maior na rotina das clínicas e consultórios (Ortega et al. 2020).

O Covid -I9 têm causado desestabilidade em todas as áreas em todo o mundo, abalo econômico, político, educacional e social. A saúde pública e privada vive um verdadeiro caos, pessoas vivendo em pânico com alto índice de estresse e ansiedade, famílias estão sendo devastadas por perdas irreparáveis de entes queridos e amigos (Dziedzic 2020).

Com a pandemia, os setores públicos e privados, tiveram que se reinventar, as plataformas virtuais foram poderosas aliadas para o desenvolvimentos das atividades profissionais e educacionais em "home office". Em contra partida, o isolamento social contribuiu para o sofrimento emocional e desenvolvimento de doenças préexistentes(Ammar et al. 2020). 
Vergara-Buenaventuraet.al. (2020) (Vergara-Buenaventura, Chavez-Tuñon, and Castro-Ruiz 2020) relatam que estratégias de suspender as atividades diárias para manter o isolamento social, associado ao medo, podem causar nos profissionais de saúde, frustrações, mau-humor, ansiedades, insônia, má qualidade nos atendimentos, transtornos, depressão, vícios e até suicídios.

Segundo Diaz et.al. (202I) (Diaz et al. 202I) todo o contexto de mudanças de vida por causa da pandemia do Covid-ı́, causou um abalo na saúde mental, gerando medos, pânicos, esgotamento, angústias e ansiedades descontroladas em pacientes, dentistas e equipe.

Um artigo publicado em julho 2020, por Gasparro et.al. (Gasparro et al. 2020) descrevem que uma pesquisa realizada na Itália com 356 dentistas, desde o início da pandemia do Covid-ı, 92\% dos pacientes cancelaram os futuros atendimentos odontológicos, com medo de serem infectados. $O$ autor ainda complementa, que as incertezas da profissão em meio ao caos pandêmico, não somente desestrutura a vida financeira, mas gera problemas psicofisiológicos.

Corroborando com os autores anteriores, Salehiniya e Abbaszadeh (2021) (Salehiniya and Abbaszadeh 2021) ainda descrevem, que além da preocupação com a diminuição da renda financeira, o profissional teme com a ausência de um tratamento específico de cura para a doença Covid-ı.

Em 2007 Bruce S. McEwen (McEwen 2007) publicou um artigo com o título Fisiologia e Neurobiologia do Stress e Adaptação: Papel Central do Cérebro, em síntese o autor descreve que o estresse colabora no processo de envelhecimento, e com o desenvolvimento de doenças como diabetes, doenças cardíacas, queda da imunidade e depressão. E para melhorar a condição da saúde mental, o autor relata que as atividades físicas são estratégias poderosas de prevenção.

Os DDS, dentro do âmbito de trabalho e das atribuições que executam, estão pautados por meio do conhecimento oferecido pelas pesquisas realizadas e protocolos estabelecidos, no que deve ser realizado e como proceder nos tratamentos odontológicos. Seguir estas orientações de fato é a priori neste momento, informar e estabelecer critérios mútuos de controle juntamente com os pacientes e equipe irá amenizar o estresse e evitar prováveis pânicos (Gugnani et al. 2020). 


\section{METODOLOGIA}

Esta revisão de literatura realizou buscas na plataforma do Pubmed, por artigos publicados até agosto de 202I referentes à pandemia Covid-19 e as consequências psicológicas que este cenário causou ao cirurgião dentista.

\section{IMPACTOS NA SAÚDE MENTAL DE DESASTRES ANTERIORES}

Pandemias anteriores causaram efeitos nocivos ao longo da história da humanidade, contribuindo para o desenvolvimento de doenças psicológicas e psicossociais (Lyons et al. 2020; Chew et al. 2020).

O distanciamento social e medidas de proteção individual, são protocolos adotados desde as pandemias anteriores (Jester, Uyeki, and Jernigan 2020). Uma das características comum entre pandemias é a disseminação do agente patógeno pela mobilidade das pessoas pelo mundo (Akin and Gözel 2020).

O isolamento social é indicado como uma forma de conter esta disseminação global (Tang et al. 2020). Mas em contrapartida esse confinamento causa impactos emocionais como: raiva, confusão mental, sofrimento, tédio, ansiedade, medos, depressão e até suicídio (Murata et al. 202I).

Chew et.al. (2020) (Chew et al. 2020) ressaltam que a busca por estratégias de sobrevivência mediante a desastres produz sentimentos positivos, diminuindo a probabilidade de desenvolver doenças psicológicas. Brookset.al (2020) (Brooks S K et al. 2020) descrevem que, as autoridades devem justificar e esclarecer a população os planos de contingência, pois a clareza das informações auxiliam na aceitação das estratégias impostas.

A ansiedade tem atingido a população de um modo geral. Esta alteração emocional pode ser considerada positiva quando promove ao indivíduo ações de enfrentamento aos eventos trágicos e/ou situações desagradáveis em sua vida, chamada está de ansiedade adaptativa. Mas quando exagerada, causa desequilíbrio e descontroles mentais e físicos, podendo ser por muitas vezes, a ansiedade exagerada mas nociva do que os problemas existentes (Dennis, Radnitz, and Wheaton 2021).

Considerando os últimos roo anos a pandemia do coranavírus 2019 é uma das maiores tragédias a saúde pública global (Chakraborty and Maity 2020). A comunidade de pesquisadores científicos tiveram grande importância no combate a esta doença, foram os 
responsáveis pelo desenvolvimento de protocolos medicamentosos ao combate dos sinais e sintomas do covid-ı9 e pela criação de vacinas para prevenção deste patógeno (Scarpa et al. 2020).

\section{PANDEMIAS QUE ENTRARAM PARA A HISTÓRIA}

Peste Negra: Teve o primeiro relato de pandemia em 54I, conhecida como praga de Justiniano, seu segundo episódio foi entre 1347 a 1352, onde cerca de um terço da população européia foi erradicada neste período. A terceira onda desta pandemia teve início em Cantão e Hong Kong em i894. Seu agente transmissor eram pulgas presentes em ratos pretos (Zietz and Dunkelberg 2004).

Praga de Justiniano: A aconteceu no ano de 54I, mas perdurou entre os séculos VI ao VIII (Cohn 2008). Segundo Raoult et.al. (2013) (Raoult et al. 2013) o imperador Justiniano I contraiu a doença, mas sobreviveu à pandemia que levou seu nome, chegou a causar milhares de mortes por dia, hoje em dia ainda há surtos da doença.

Varíola: Considerada umas das doenças mais devastadoras da humanidade, aparece nos primeiros escritos egípcios, indianos e chineses. Estima-se que no final do século XVIII, a varíola matou cerca de 400.000 pessoas a cada ano. Devido à campanha de vacinação, tornou-se raro os surtos de varíola desde 1980 (Duggan et al. 2016).

Influenza A: $\mathrm{O}$ vírus da influenza A causou três episódios pandêmicos ao longo do século XX (Kong et al. 2020), onde impactou a vida da população mundial.

- Em I9I8 (Spanish flu - HINI) causando 50 milhões de mortes;

- Em 1957 (Asian flu - $\mathrm{H}_{2} \mathrm{~N}_{2}$ ) que levou a óbito cerca de I milhão de pessoas;

- Em 1968 (Hong Kong flu - $\mathrm{H}_{3} \mathrm{~N}_{2}$ ) registrando a média de 2,5 milhões de mortes.

Gripe suína: Causada pelo vírus influenza $\mathrm{A} \mathrm{H}_{\mathrm{I}} \mathrm{N}$, a gripe suína teve as primeiras manifestações em humanos no ano de 2009 no México. Sua predileção eram adultos jovens e os sintomas se apresentavam de forma branda (Mena et al. 20r6).

Tifo: Trata-se de uma doença da antiguidade, em 1546 o médico Fracastorius descreve o tifo no tratado sobre doenças infecciosas. Relatos apontam o pior surto de tifo descrito na história entre os anos de 1917 a 1925, neste período cerca de 3 milhões de russos morreram (Raoult, Woodward, and Dumler 2004).

Cólera: Foram registrados 7 pandemias da cólera, a primeira foi no ano de 1817 e a última registra em 196I, com surtos periódicos até os dias atuais (Deen, Mengel, and 
Clemens 2020). Segundo a OMS a cólera é a causadora da morte de 3 a 5 milhões de pessoas por ano, sendo um problema de saúde pública mundial, principalmente nos países asiáticos e africanos (Harris et al. 2012).

Tuberculose: Desde a antiguidade a tuberculose é relatada, segundo Barberis et. al. (2017) (Barberis et al. 2017) os primeiros escritos sobre a doença apareceram na China e Índia a mais de 3.000 anos atrás. A primeira contaminação em massa foi registrado em i6oo na Europa (Bates and Stead i993).

Aids: Pandemia de longa duração, a aids teve sua origem na África por volta de 1920 (Faria et al. 2014). No ano de 198I, o HIV se espalhou, infectando muitas pessoas, isto levou o despertamento da comunidade cientista em investigar o vírus. Após 40 anos acompanhando está doença, os registros mostram que cerca de 38 milhões de pessoas em todo o mundo convivem com o vírus HIV e aproximamente 32 milhões da população mundial já morreram. Até os dias atuais a medicina não encontrou a cura da doença, somente tratamento de controle e prevenção (de Cock, Jaffe, and Curran 202I).

Ebola: Doença altamente virolenta, o ebola é responsável por causar muitas mortes, sua taxa de letalidade é em média 50\%. Sua origem foi na África em 1976, desde então foram descritos 3i surtos de ebola no continente africano, o último iniciou-se em junho de 2020 e está em andamento (Batra et al. 2020).

\section{IMPACTO DA PANDEMIA COVID-ı NA VIDA DO CIRURGIÃO DENTISTA}

As condições atuais da pandemia covid-ıg são favoráveis para as alterações emocionais, é relevante neste momento prestarmos atenção em nós mesmos, pois algumas dessas emoções dentro de um contexto de desordem, podem desencadear transtornos psíquicos e/ou fisiológicos (Usher, Durkin, and Bhullar 2020; Dubey et al. 2020).

Com a explosão da pandemia coronavírus, o desequilíbrio mental foi consideravelmente potencializado. Os DDS tiveram suas práticas clínicas odontológicas reduzidas por conta do alto risco de infecção, os procedimentos ambulatoriais se restringiram somente aos casos emergenciais, isto contrubuiu para um novo problema, a diminuição da renda familiar financeira de muitos dentistas (Açikgöz and Günay 2020).

A rotina dos profissionais liberais que antes eram tratar vários pacientes ao longo do dia, apresentou grande redução. Uma pesquisa feita no norte da Itália por Consolo et.al. (2020) (Consolo et al., n.d.) entrevistaram 356 dentistas proprietários de consultórios 
privados, 70\% relataram que antes do início da pandemia atendiam de 6 a 15 pacientes por dia e que atualmente os atendimentos se limitam de o a 5 pacientes por semana.

Além da adaptação a uma renda mensal reduzida, o isolamento social foi instaurado (Banerjee and Rai 2020). Alvares et.al. (202I) (Alvares et al. 202I) realizaram um estudo no Brasil com r.6o9 profissionais da saúde para avaliar os transtornos mentais causado pela pandemia atual. Entre os voluntários haviam dentistas, médicos, enfermeiros, nutricionistas, psicólogos, farmacêuticos, fisioterapeutas e outros. Os resultados apontaram que os dentistas foram os que mais permaneceram em isolamento domiciliar por motivo de suspensão das atividades profissionais. $O$ desequilibrio mental foi demonstrado em $90 \%$ de todos os entrevistados.

A convivência familiar aumentou e muitos se depararam com rotinas caseiras e afazeres domésticos. Adequações tiveram que ser adotadas para que todos tivessem um ambiente sadio, afim de evitar conflitos e não potencializar os abalos emocionais que a situação atual proporciona (Jakovljevic et al. 2020).

Barabari (2020) (Barabari and Moharamzadeh 2020) ressalta que a pandemia Covid19 não só prejudicou os DDS, mas também os estudantes de odontologia. No ambiente acadêmico uma parcela de alunos sofrem em estar confinados em casa, recebendo os conteúdos teóricos em formato virtual. Enquanto outros apresentam medos e angústias em continuar atendendo os pacientes na clínica escola, tendo que procurar ajuda psicológica para continuar sua graduação.

Brookset.al (2020) (Brooks S K et al. 2020) abordam que as medidas de enfrentamento a tragédias impostas pelas autoridades, devem considerar não somente o benefício do isolamento social que é resguardar a saúde física, mas também desenvolver estratégias para conter as doenças psicológicas que a quarentena desencadeia.

Ahmed et.al. (2020) (Ahmed et al. 2020) realizaram uma pesquisa com 669 dentistas de 30 países diferentes, avaliando o impacto emocional em meio a pandemia covid-i9. Os resultados mostraram que cerca de $90 \%$ dos entrevistados afirmaram ter medo e ansiedade quando seus pacientes apresentam tosse ou suspeita de infecção e relataram também o medo de transportarem o covid-19 pras suas casas. Cerca de $75 \%$ declararam o medo de ficarem em isolamento por infecção e medo dos custos dos tratamentos. O percentual de $66 \%$ confessaram o desejo de suspender os atendimentos odontológicos até que os casos de coranovírus diminua. 
$\mathrm{Na}$ Alemanha pesquisas para avaliar o impacto emocional foram conduzidas pelos cientistas Mekhemar et.al. (202I) (Mekhemar et al. 202I), 732 dentistas foram entrevistados, 95\% eram proprietários de consultórios privados. Em síntese as mulheres entrevistadas apresentaram maior grau de medo, ansiedade e estresse comparado aos homens.

$\mathrm{Na}$ China Tao et.al. (202I) (Tao et al. 2020) realizou um estudo com 969 dentistas, os transtornos psicológicos descritos foram ansiedade, depressão, medos, impotência e pânico de serem infectados. Observou-se que os dentistas que possuiam doenças préexistentes apresentaram maior índice de descontrole emocional.

$\mathrm{Na}$ Noruega I.237 voluntários composto por dentistas e equipe participaram de um estudo (Uhlen, Ansteinsson, and Korzeniewska 202I). A maioria apresentou alterações emocionais relacionada ao medo de infecção de si próprio e familiares. Os resultados demonstratam que profissionais com maior experiência de trabalho, possuem maior equilíbrio emocional comparado aos profissionais com poucos anos de atuação.

No Reino Unido mais de 5.000 voluntários dentistas foram avaliados, 77\% destes profissionais relataram que a pandemia afetou a vida financeira e este foi o fator que mais causou angústias, medos e preocupações (Collin, Selmo, and Whitehead 202I).

É primordial que o DDS mantenha sua saúde mental, sendo resiliente mediante as situações atuais, pois somente assim sera possível elaborar um planejamento útil para se manter ativo na profissão (Bonanno 2004).

O foco é encontrar soluções para o enfrentamento a pandemia do coronavírus, afim de preservar a integridade da saúde física e mental dos profissionais dentistas, familiares e de seus pacientes (Nicola et al. 2020).

\section{ESTRATÉGIAS E RECOMENDAÇÕES}

Embora pesquisas sobre a fisiopatologia do novo Coronavírus (SARS-Cov-2) sejam de crucial importância na busca de tratamento e métodos de prevenção para COVID 19, observa-se a valorização dos aspectos biológicos por parte dos estudos em detrimento das implicações psicológicas da doença, possibilitando, o surgimento de sintomas psicopatológicos e doenças psiquiátricas associadas (Shuja et al. 2020).

Neste sentido, existem recomendações para profissionais de saúde que lidam diretamente com pacientes e que representam grupo de risco para contágio por 
Coronavírus. As medidas de prevenção englobam principalmente reconhecimento de emoções como ansiedade, medo e sinais de esgotamento físico e mental. Algumas dessas recomendações podem ser descritas como:

- Aceitar o distanciamento social como medida necessária de proteção para si próprio e sua família (Babore et al. 2020);

- Persistir em pensamentos e atitudes positivas, e oferecer apoio quando possível, isto desenvolve um ambiente menos estressante e mais produtivo (Babore et al. 2020; Giorgi et al., n.d.);

- Contar com o apoio da familia é um poderoso fator de proteção a traumas e causa bem-estar mental (Jav 2020);

- Autocuidado, reconhecer sintomas de estresse (irritação, nervosismos, falta de motivação, cansaço, entre outros) e buscar apoio psicológico (Jav 2020);

- Identificar estratégias individuais (atividades de lazer que causam sensação de bem estar), buscando praticá-las regularmente. Alguns exemplos: ler, ouvir música, oração, meditação, culinária, entre outros ( $\mathrm{Nu}$ et al. 2020);

- Evitar estratégias consideradas inúteis: uso de substâncias psicoativas, tabagismo e uso excessivo do álcool ( $\mathrm{Nu}$ et al. 2020);

- Manter rotina saudável: sono adequado, boa alimentação e prática de atividade física que considere prazerosa ( $\mathrm{Nu}$ et al. 2020).

Ornell, et al. (2020) (Ornell et al. 2020) alertam sobre a necessidade da implementação de ações estratégicas por parte de órgãos governamentais a fim de minimizar sintomas psicopatológicos presentes em situações de pandemia e desastres de grande escala. Alguns exemplos são descritos a seguir:

- Realizar treinamento para profissionais da saúde sobre gerenciamento de estresse, trauma, depressão e comportamento de risco;

- Fornecer canais oficiais para obtenção de informações atualizadas ao público sobre tratamentos e medidas de prevenção;

- Monitorar e refutar notícias falsas;

- Garantir recursos e infraestrutura adequados para disponibilizar serviços de saúde mental;

- Desenvolver materiais psicoeducacionais de fácil acesso; 
- Incentivar pesquisas científicas;

- Considerar e respeitar fatores culturais na implementação de políticas públicas.

Importante ressaltar que a forma como cada indivíduo responde ao estresse causado pela pandemia de COVID 19 depende de fatores como: gênero, apoio social, situação financeira, condições de saúde, recursos presentes na comunidade em que vive, entre outros (Torales et al. 2020).

Por fim, aos profissionais de saúde cabe ressaltar a importância da aceitação dos limites de sua atuação e reconhecimento do papel crucial que desempenham no combate à pandemia de COVID ig.

\section{CONCLUSÃO}

Em meio ao cenário catastrófico de infecções pelo mundo, tudo ficou muito incerto ao profissional da odontologia. Os DDS do mundo tiveram suas vidas afetadas pela pandemia Covid-ı9. Tornou-se necessário a adaptação na redução da jornada de trabalho e em consequência disso a diminuição da renda financeira. Além disso, muitos profissionais viveram o luto pela morte de familiares, foi sem dúvida o pior ano da vida de muitos profissionais, cercados de tristezas, medos e falta de perspectivas.

A saúde mental é fundamental para que o DDS possa continuar exercendo sua profissão de forma satisfatória e com excelência. Para isso, é importante o autocuidado, buscar realizar atividades que lhe tragam prazer; aceitar as novas condições de trabalho com resiliência, estar sempre apoiando a equipe de trabalho e contar com o apoio dos familiares. Estes são sem dúvida, poderosos fatores de proteção ao desequilíbrio mental.

Vale ressaltar a importância de um apoio psicológico profissional, isto trará liberdade para expor os medos, angústias e preocupações. O equilíbrio emocional é fundamental para estabelecer estratégias úteis e elaborar planejamentos futuros para a rotina familiar e profissional.

Este estudo apresenta limitações por existem poucos artigos publicados avaliando o impacto psicológico em DDS com práticas em consultórios privados.

\section{REFERÊNCIAS}

Açikgöz, Ömer, and Aslı Günay. 2020. "The Early Impact of the Covid-ı9 Pandemic on the Global and Turkish Economy." Turkish Journal of Medical Sciences 50 (SI-I): 520-26. https://doi.org/ro.3906/sag-2004-6. 
Ahmed, Muhammad Adeel, Rizwan Jouhar, Naseer Ahmed, Samira Adnan, Marziya Aftab, Muhammad Sohail Zafar, and Zohaib Khurshid. 2020. "Fear and Practice Modifications among Dentists to Combat Novel Coronavirus Disease ( COVID-19) Outbreak."

Akin, Levent, and Mustafa Gökhan Gözel. 2020. "Understanding Dynamics of Pandemics." Turkish Journal of Medical Sciences 50 (SI-I): 515-19. https://doi.org/ro.3906/sag-2004-133.

Alvares, Juliana, Duarte Bonini, Campos Bianca, Gonzalez Martins, and Lucas Arrais. 2021. "Symptoms Related to Mental Disorder in Healthcare Workers during the COVID 19 Pandemic in Brazil." International Archives of Occupational and Environmental Health, no. o123456789. https://doi.org/10.1007/s00420-021-01656-4.

Amato, Alessandra, Mario Caggiano, Massimo Amato, Giuseppina Moccia, Mario Capunzo, and Francesco De Caro. 2020. "Infection Control in Dental Practice during the Covid-19 Pandemic." International Journal of Environmental Research and Public Health 17 (13): I-I2. https://doi.org/Io.3390/ijerphi7134769.

Ammar, Achraf, Patrick Mueller, Khaled Trabelsi, Hamdi Chtourou, Omar Boukhris, Liwa Masmoudi, Bassem Bouaziz, et al. 2020. "Psychological Consequences of COVID-I9 Home Confinement: The ECLB-COVIDig Multicenter Study.” PLoS ONE I5 (II): I-I3. https://doi.org/ro.1371/journal.pone.0240204.

Babore, Alessandra, Lucia Lombardi, Maria Luisa Viceconti, Silvia Pignataro, Valentina Marino, Monia Crudele, Carla Candelori, Sonia Monique Bramanti, and Carmen Trumello. 2020. "Psychological e Ff Ects of the COVID-2019 Pandemic: Perceived Stress and Coping Strategies among Healthcare Professionals." Psychiatry Research 293 (May): II3366. https://doi.org/ı.ıor6/j.psychres.2020.113366.

Banerjee, Debanjan, and Mayank Rai. 2020. "Social Isolation in Covid-19: The Impact of Loneliness." International Journal of Social Psychiatry 66 (6): 525-27. https://doi.org/10.1177/0020764020922269.

Barabari, Poyan, and Keyvan Moharamzadeh. 2020. "Novel Coronavirus (Covid-I9) and Dentistry-a Comprehensive Review of Literature." Dentistry Journal 8 (2): I-I8. https://doi.org/ro.339o/dj8020053.

Barberis, I., N. L. Bragazzi, L. Galluzzo, and M. Martini. 2017. "The History of Tuberculosis: From the First Historical Records to the Isolation of Koch's Bacillus." Journal of Preventive Medicine and Hygiene 58 (I): E9-I2. https://doi.org/I0.15167/24214248/jpmh2017.58.1.728.

Bates, J. H., and W. W. Stead. 1993. "The History of Tuberculosis as a Global Epidemic." The Medical Clinics of North America 77 (6): 1205-17. https://doi.org/10.1016/Soo257125(16)30188-2. 
Batra, Simran, Rohan Kumar Ochani, Mufaddal Najmuddin Diwan, Farah Yasmin, Suha Safi Qureshi, Sameer Bhimani, Shehryar Shaikh, et al. 2020. "Clinical Aspects of Ebola Virus Disease: A Review." Infezioni in Medicina 28 (2): 212-22.

Bonanno, George A. 2004. "Loss, Trauma, and Human Resilience: Have We Underestimated the Human Capacity to Thrive after Extremely Aversive Events?" American Psychologist 59 (I): 20-28. https://doi.org/10.1037/ooo3-066X.59.1.20.

Brooks S K, Webster R K, Smith L E, Woodland L, Wessely S, Greenberg N, and Rubin G J. 2020. "The Psychological Impact of Quarantine and How to Reduce It: Rapid Review of the Evidence [Revista En Internet] 2020; 395(I0227): 912-920.” The Lancet 395 (I0227): 9122o. https://www.ncbi.nlm.nih.gov/pmc/articles/PMC 7158942/pdf/main.pdf.

Bryant-genevier, Jonathan, Carol Y Rao, Barbara Lopes-cardozo, Ahoua Kone, and Charles Rose. 2021. "Symptoms of Depression, Anxiety, Post-Traumatic Stress Disorder , and Suicidal Ideation Among State, Tribal, Local, and Territorial Public Health Workers During the COVID-19 Pandemic - United States, March - April 2021" 70 (26): 947-52.

Buselli, Rodolfo, Martina Corsi, Sigrid Baldanzi, Martina Chiumiento, Elena Del Lupo, Valerio Dell Oste, Carlo Antonio Bertelloni, et al. n.d. "Professional Quality of Life and Mental Health Outcomes among Health Care Workers Exposed To" 2 (December 2019): I-I2.

Chakraborty, Indranil, and Prasenjit Maity. 2020. "Science of the Total Environment COVID-i9 Outbreak: Migration, Effects on Society, Global Environment and Prevention." Science of the Total Environment 728: 138882. https://doi.org/Io.1016/j.scitotenv.2020.138882.

Chew, Qian Hui, Ker Chiah Wei, Shawn Vasoo, Hong Choon Chua, and Kang Sim. 2020. "Narrative Synthesis of Psychological and Coping Responses towards Emerging Infectious Disease Outbreaks in the General Population : Practical Considerations for the COVID-19 Pandemic" 6r (7): 350-56.

Cock, Kevin M. de, Harold W. Jaffe, and James W. Curran. 2021. "Reflections on 40 Years of AIDS." Emerging Infectious Diseases 27 (6): 1553-60. https://doi.org/10.3201/eid2706.210284.

Cohn, Samuel K. 2008. "Epidemiology of the Black Death and Successive Waves of Plague." Medical History, no. SUPPL. 27: 74-1oo. https://doi.org/I0.1017/s0025727300072100.

Collin, Victoria, Ellena O Selmo, and Penny Whitehead. 2021. "Psychological Distress and the Perceived Impact of the COVID-ig Pandemic on UK Dentists during a National Lockdown,” I-8.

Consolo, Ugo, Pierantonio Bellini, Davide Bencivenni, Cristina Iani, and Vittorio 
Checchi. n.d. "Epidemiological Aspects and Psychological Reactions to COVID-I9 of Dental Practitioners in the Northern Italy Districts of Modena and Reggio Emilia."

Coulthard, Paul. 2020. "Dentistry and Coronavirus (COVID-19) - Moral DecisionMaking.” British Dental Journal 228 (7): 503-5. https://doi.org/I0.1038/s41415-020-I482-I.

Deen, Jacqueline, Martin A. Mengel, and John D. Clemens. 2020. "Epidemiology of Cholera." Vaccine 38: A31-40. https://doi.org/Io.IoI6/j.vaccine.2019.07.078.

Dennis, Danielle, Cynthia Radnitz, and Michael G. Wheaton. 2021. "Correction to: A Perfect Storm? Health Anxiety, Contamination Fears, and COVID-19: Lessons Learned from Past Pandemics and Current Challenges (International Journal of Cognitive Therapy, (2021), 10.1007/S41811-02I-00109-7)." International Journal of Cognitive Therapy. https://doi.org/Io.I007/s4I8II-02I-oolio-o.

Derruau, Stéphane, Jérôme Bouchet, Ali Nassif, Alexandre Baudet, Kazutoyo Yasukawa, Sandrine Lorimier, Isabelle Prêcheur, et al. 2021. "COVID-I9 and Dentistry in 72 Questions: An Overview of the Literature." Journal of Clinical Medicine ro (4): 779. https://doi.org/10.3390/jcmioo40779.

Diaz, María Carrillo, Laura Lacomba Trejo, Valle González, Martín Romero Maroto, María José, and González Olmo. 202r. "Anxiety and Facial Self - Contacts : Possible Impact on COVID - 19 Transmission in Dental Practice." BMC Oral Health, I-9. https://doi.org/Io.1186/s12903-021-01564-6.

Dubey, Souvik, Payel Biswas, Ritwik Ghosh, Subhankar Chatterjee, Mahua Jana, Subham Chatterjee, Durjoy Lahiri, and Carl J Lavie. 2020. "Diabetes \& Metabolic Syndrome: Clinical Research \& Reviews Psychosocial Impact of COVID-19.” Diabetes or Metabolic Syndrome: Clinical Research or Reviews $14 \quad$ (5): 779-88. https://doi.org/ıo.1016/j.dsx.2020.05.035.

Duggan, Ana T., Maria F. Perdomo, Dario Piombino-Mascali, Stephanie Marciniak, Debi Poinar, Matthew V. Emery, Jan P. Buchmann, et al. 2016. "I7th Century Variola Virus Reveals the Recent History of Smallpox." Current Biology 26 (24): 3407-12. https://doi.org/ro.ıor6/j.cub.2016.10.o6r.

Dziedzic, Arkadiusz. 2020. "Special Care Dentistry and Covid-19 Outbreak: What Lesson Should We Learn?” Dentistry Journal 8 (2): 10-I2. https://doi.org/ı0.3390/dj8020046.

Faria, Nuno R, Andrew Rambaut, Marc A Suchard, Guy Baele, Trevor Bedford, Melissa J Ward, Andrew J Tatem, et al. 2014. "The Early Spread and Epidemic Ignition of HIV-I in Human Populations" 346 (6205).

Gasparro, Roberta, Cristiano Scandurra, Nelson Mauro Maldonato, Pasquale Dolce, Vincenzo Bochicchio, Alessandra Valletta, Gilberto Sammartino, et al. 2020. "Perceived Job Insecurity and Depressive Symptoms among Italian Dentists: The Moderating Role of Fear of Covid-19." International Journal of Environmental Research and Public Health 17 (I5): I-I2. https://doi.org/10.339o/ijerphi7155338. 
Giorgi, Gabriele, Luigi Isaia Lecca, Federico Alessio, Georgia Libera Finstad, Giorgia Bondanini, Lucrezia Ginevra Lulli, Giulio Arcangeli, and Nicola Mucci. n.d. "COVID-I9Related Mental Health E Ff Ects in the Workplace: A Narrative Review," no. December 2019: I-22.

Gugnani, Neeraj, Shalini Gugnani, B Shay, A Kolokythas, and G Almoznino. 2020. "Safety Protocols for Dental Practices in the COVID-I9 Era," 56-57. https://doi.org/10.1177/o022034520920580.2.

Harris, JB, RC LaRocque, F Qadri, ET Ryan, and SB Calderwood. 2012. "NIH Public Access - Cholera." The Lancet 379 (9835): 22. https://doi.org/ro.1or6/Sor40-6736(12)6o436X.Cholera.

Innes, N, I G Johnson, W Al-yaseen, R Harris, R Jones, S Kc, and S Mcgregor. 2020. "Since January 2020 Elsevier Has Created a COVID-I9 Resource Centre with Free Information in English and Mandarin on the Novel Coronavirus COVID- I9 . The COVID-ig Resource Centre Is Hosted on Elsevier Connect, the Company's Public News and Information," no. January.

Jakovljevic, Miro, Sarah Bjedov, Nenad Jaksic, and Ivan Jakovljevic. 2020. "Covid-19 Pandemia and Public and Global Mental Health from the Perspective of Global Health Security.” Psychiatria Danubina 32 (I): 6-I4. https://doi.org/I0.24869/psyd.202o.6.

Jav, Alena. 2020. "Since January 2020 Elsevier Has Created a COVID-I9 Resource Centre with Free Information in English and Mandarin on the Novel Coronavirus COVID- I9 . The COVID-ig Resource Centre Is Hosted on Elsevier Connect, the Company's Public News and Information ," no. January.

Jester, Barbara J., Timothy M. Uyeki, and Daniel B. Jernigan. 2020. "Fifty Years of Influenza $\mathrm{A}\left(\mathrm{H}_{3} \mathrm{~N}_{2}\right)$ Following the Pandemic of 1968." American Journal of Public Health ino (5): 669-76. https://doi.org/10.2105/AJPH.2019.305557.

Khan, Merajuddin, and Shams T Khan. 2021. "Epidemiology and Progress So Far," I-25.

Kong, Hong, General Practitioners, U K Government, Hong Kong, Soviet Union, and Hong Kong. 2020. "Perspectives The Art of Medicine Revisiting the 1957 and 1968 Influenza Pandemics" 6736 (20): 2020-22. https://doi.org/ro.1016/Sor40-6736(20)31201-0.

Levit, Michael, and Leah Levit. 2021. "Infection Risk of COVID-19 in Dentistry Remains Unknown: A Preliminary Systematic Review." Infectious Diseases in Clinical Practice 29 (2): e70-77. https://doi.org/I0.1097/ipc.0oooooooooooo939.

Lyons, D., M. Frampton, S. Naqvi, D. Donohoe, G. Adams, and K. Glynn. 2020. "Fallout from the COVID-i9 Pandemic-Should We Prepare for a Tsunami of Post Viral Depression?" Irish Journal of Psychological Medicine 37 (4): 295-300. https://doi.org/Io.1017/ipm.2020.40. 
Marqu, Montse, and L Domingo. 2021. "Contamination of Inert Surfaces by SARS-CoV2: Persistence, Stability and Infectivity . A Review" 193 (December 2020). https://doi.org/Io.1o16/j.envres.2020.110559.

Maunder, Robert. 2004. "The Experience of the 2003 SARS Outbreak as a Traumatic Stress among Frontline Healthcare Workers in Toronto: Lessons Learned." Philosophical Transactions of the Royal Society B: Biological Sciences 359 (I447): III7-25. https://doi.org/10.1098/rstb.2004.1483.

McEwen, Bruce S. 2007. "Physiology and Neurobiology of Stress and Adaptation: Central Role of the Brain." Physiological Reviews 87 (3): 873-904. https://doi.org/I0.1152/physrev.00041.2006.

Mekhemar, Mohamed, Sameh Attia, Christof Dörfer, and Jonas Conrad. 2021. "The Psychological Impact of the COVID-ı9 Pandemic on Dentists in Germany."

Mena, Ignacio, Martha I. Nelson, Francisco Quezada-Monroy, Jayeeta Dutta, Refugio Cortes-Fernández, J. Horacio Lara-Puente, Felipa Castro-Peralta, et al. 2016. "Origins of the $2009 \mathrm{HIN}_{\mathrm{I}}$ Influenza Pandemic in Swine in Mexico." ELife 5 (JUN2016): I-2I. https://doi.org/ro.7554/eLife.16777.

Meyerowitz, Eric A., Aaron Richterman, Rajesh T. Gandhi, and Paul E. Sax. 202I. "Transmission of SARS-CoV-2: A Review of Viral, Host, and Environmental Factors." Annals of Internal Medicine 174 (I): 69-79. https://doi.org/10.7326/M20-5008.

Mulugeta, Tewodros, Elazar Tadesse, Tewodros Shegute, and Takele Taye Desta. 202I. "COVID-19: Socio-Economic Impacts and Challenges in the Working Group." Heliyon 7 (6). https://doi.org/ı.1016/j.heliyon.2021.e07307.

Murata, Stephen, Taylor Rezeppa, Brian Thoma, Laura Marengo, Katie Krancevich, Elizabeth Chiyka, Benjamin Hayes, et al. 2021. "The Psychiatric Sequelae of the COVID19 Pandemic in Adolescents, Adults, and Health Care Workers.” Depression and Anxiety 38 (2): 233-46. https://doi.org/10.1002/da.23120.

Nemeth, Orsolya, Mercedesz Orsos, Fanni Simon, and Peter Gaal. 2021. "An Experience of Public Dental Care during the COVID-ig Pandemic : Reflection and Analysis."

Nicola, Maria, Zaid Alsafi, Catrin Sohrabi, Ahmed Kerwan, Ahmed Al-jabir, Christos Iosifidis, Maliha Agha, and Riaz Agha. 2020. "The Socio-Economic Implications of the Coronavirus Pandemic ( COVID-19): A Review." International Journal of Surgery 78 (March): 185-93. https://doi.org/10.1or6/j.ijsu.2020.04.or8.

$\mathrm{Nu}$, Mila, Nu Htay, Roy Rillera, Rafidah Bahari, Ayesha Alrifai, and Fatjona Kamberi. 2020. "Since January 2020 Elsevier Has Created a COVID-19 Resource Centre with Free Information in English and Mandarin on the Novel Coronavirus COVID- I9 . The COVID-i9 Resource Centre Is Hosted on Elsevier Connect, the Company's Public 
News and Information," no. January.

Ornell, Felipe, Jaqueline B Schuch, Anne O Sordi, Felix Henrique, and Paim Kessler. 2020. ““" Pandemic Fear "” and COVID-19: Mental Health Burden and Strategies” 42 (3): 232-35. https://doi.org/10.1590/1516-4446-2020-0008.

Ortega, Karem L, Alessandra Rodrigues De Camargo, Juliana Bertoldi Franco, Antonio Mano Azul, Mario Pérez Sayáns, Paulo Henrique, and Braz Silva. 2020. "SARS-CoV-2 and Dentistry," Io-II.

Passarelli, Pier Carmine, Edoardo Rella, Paolo Francesco Manicone, Franklin GarciaGodoy, and Antonio D'Addona. 2020. "The Impact of the COVID-I9 Infection in Dentistry." Experimental Biology and Medicine 245 (II): 940-44. https://doi.org/ıo.1177/1535370220928905.

Raoult, Didier, Nadjet Mouffok, Idir Bitam, Renaud Piarroux, and Michel Drancourt. 2013. "Plague: History and Contemporary Analysis." Journal of Infection 66 (I): 18-26. https://doi.org/10.1or6/j.jinf.2012.09.o10.

Raoult, Didier, Theodore Woodward, and J. Stephen Dumler. 2004. "The History of Epidemic Typhus." Infectious Disease Clinics of North America I8 (I): I27-40. https://doi.org/ro.1016/So891-5520(03)0oo93-X.

Salehiniya, Hamid, and Hamid Abbaszadeh. 2021. "Prevalence of Corona-Associated Anxiety and Mental Health Disorder among Dentists during the COVID-r9 Pandemic." Neuropsychopharmacology Reports, no. March: I-7. https://doi.org/I0.1002/npr2.12179.

Scarpa, Raffaele, Francesco Caso, Luisa Costa, Saverio Passavanti, Maria Grazia Vitale, Claudia Trojaniello, Antonio Del Puente, and Paolo A. Ascierto. 2020. "May the Analysis of 1918 Influenza Pandemic Give Hints to Imagine the Possible Magnitude of Corona Virus Disease-2019 (COVID-19)?” Journal of Translational Medicine I8 (I): I-I2. https://doi.org/ı.1186/si2967-020-02673-6.

Shuja, Kanwar Hamza, Muhammad Aqeel, Abbas Jaffar, and Ammar Ahmed. 2020. "COVID-I9 PANDEMIC AND IMPENDING GLOBAL MENTAL HEALTH IMPLICATIONS” 32 (I): 32-35.

Tang, Biao, Fan Xia, Sanyi Tang, Nicola Luigi, and Qian Li. 2020. "Since January 2020 Elsevier Has Created a COVID-I9 Resource Centre with Free Information in English and Mandarin on the Novel Coronavirus COVID- 19. The COVID-ig Resource Centre Is Hosted on Elsevier Connect, the Company's Public News and Information," no. January.

Tao, Jiang, Yueting Lin, Long Jiang, Zhuojun Zhou, and Junjun Zhao. 2020. "Since January 2020 Elsevier Has Created a COVID-19 Resource Centre with Free Information in English and Mandarin on the Novel Coronavirus COVID- 19 . The COVID-19 Resource Centre Is Hosted on Elsevier Connect, the Company's Public News and 
Information ," no. January.

Torales, Julio, Marcelo O Higgins, João Mauricio Castaldelli-maia, and Antonio Ventriglio. 2020. "The Outbreak of COVID-I9 Coronavirus and Its Impact on Global Mental Health,” 3-6. https://doi.org/10.1177/o020764020915212.

Uhlen, M M, V E Ansteinsson, and L Korzeniewska. 2021. "Psychological Impact of the COVID-I9 Pandemic on Dental Health Personnel in Norway," I-II.

Usher, Kim, Joanne Durkin, and Navjot Bhullar. 2020. "The COVID-19 Pandemic and Mental Health Impacts." International Journal of Mental Health Nursing 29 (3): 315-18. https://doi.org/ro.IIII/inm.12726.

Vergara-Buenaventura, Andrea, Mariella Chavez-Tuñon, and Carmen Castro-Ruiz. 2020. "The Mental Health Consequences of Coronavirus Disease 2019 Pandemic in Dentistry." Disaster Medicine and Public Health Preparedness I4 (6): e3I-34. https://doi.org/ro.1017/dmp.2020.190.

Villani, Federico Alcide, Riccardo Aiuto, Luigi Paglia, and Dino Re. 2020. "COVID-I9 and Dentistry: Prevention in Dental Practice , a Literature Review," I-I2.

Zietz, Bj^rn P, and Hartmut Dunkelberg. 2004. "The History of the Plague and the Research on the Causative Agent Yersinia Pestis” 207. 